\title{
Distance restricted maximal covering model for pharmacy duty scheduling problem
}

\author{
Nuşin Uncu ${ }^{\text {* }}$, Berna Bulğurcu ${ }^{b}$ Fatih K1lıç \\ ${ }^{a}$ Department of Industrial Engineering, Adana Science and Technology University, Turkey \\ ${ }^{b}$ Deparment of Business, Çukurova University, Turkey \\ ${ }^{c}$ Department of Computer Engineering, Adana Science and Technology University, Turkey \\ nuncu@adanabtu.edu.tr, bkiran@cu.edu.tr, fkilic@adanabtu.edu.tr
}

\section{ARTICLE INFO}

Article history:

Received: 27 October 2017

Accepted: 4 April 2018

Available Online: 1 June 2018

Keywords:

Pharmacy

Duty scheduling

Location models

Maximal covering

Distance restricted

AMS Classification 2010:

90C11, 90C 90

\begin{abstract}
Pharmacies are considered as an integral part of health care systems for supplying medicine to patients. In order to access medicine with ease, pharmacies locations in the context of distance and demand are important for patients. In the case of a few numbers of pharmacies may be on duty at nights or during holidays, pharmacies duty scheduling problem occur and can be associated with location models. In contrast to widely used $p$-median model which aims to minimize the demand-weighted distance, we maximize the demand covered over the distance between the patients and the pharmacies on duty. Main contribution of the proposed model is the restriction constraint for the distance between pharmacies on duty in order to ensure fairness in an organizational view of point. We propose a distance restricted maximal covering location model (DR-MCLM) in this study. This mathematical model is a mixed integer linear programming model and solved by Lingo optimization software. The distances between the pharmacies and the sites are obtained using Geographic Information Systems (GIS). The model is applied for the case in Adana, one of the biggest cities in Turkey. The results are given on the maps of the city, including the pharmacies on duty and their assignments to sites in each day of the period.
\end{abstract}

\section{Introduction}

Pharmacies supply medicine and medical instruments to patients. Location of pharmacies is important for patients and usually depends on population distribution over city and closeness to hospitals. Patients and their relatives need them to access medicine in the daytime and night period. In Turkey, it is forbidden to sell medicine in anywhere other than in pharmacies. Therefore, people can get medicine only from the pharmacies. This results in the need of pharmacies to be open after the daytime period and meet the urgent demands. However, not all pharmacies are allowed to work at nights. The problem of which pharmacies should be kept open and which ones closed at nights is the main concern of the duty scheduling problem of pharmacies. The Chamber of Pharmacists is responsible for pharmacies duty scheduling as considering fairness toward total number of duties given to each pharmacy. This paper aims to develope a mathematical model for the fairest optimal duty scheduling of pharmacies.

Location theory includes such models representing the scheduling and assignment problems. Set covering model is the well known and base one in the location theory. It is used to find the minimum number of facilities to cover all the demand points in a given region. Other important one is the maximal covering location model (MCLM) and it seeks to locate facilities in order to maximize the coverage of all the demand points. The remained covering models can be classified as $p$-center and $p$-median models. $P$-center model seeks to find the location of $p$ facilities to minimize the maximum distance between each facility and the demand point in the given region. The quantity of demand covered is not considered in a $p$-center model, whereas in a $p$-median model demand weighted distance minimization is taken into account. In the case of health care problems, while distance has a significant role in saving life, quantity of demand is also the other critical factor for assigning a facility to a site.

${ }^{*}$ Corresponding author 
In this study, MCLM formulated in [1] is revised for the distance restricted pharmacy duty scheduling problem. In the suggested model, in addition to the demand and the distance between pharmacies and sites, distance restricted between pharmacies is also considered.

In this paper, the introduction part is followed by a literature review with four other sections. The review provides an overview of the existing literature on the pharmacy duty scheduling problem and the maximal covering location models formulated mainly for the aforementioned problem. The third section suggests a new mathematical model constructed based on the new constraint that restricts the distance between pharmacies. In the fourth section, an application of the model is provided. The results are mapped by using geographic information system tools in the following section. Finally, in the last section we draw some conclusions and make further suggestions.

\section{Literature review}

The duty scheduling problem has been studied in a widespread area of operations research. However, it is not the same for pharmacy duty scheduling problem. In the first part of the review, the studies on the duty scheduling of pharmacies is demonstrated. In the second part, we present the related literature about the maximal covering location model which forms the mathematical basis for the suggested pharmacy duty scheduling problem in this study.

Variable neighbourhood search (VNS) is used to solve a multi period $p$-median problem with the real data obtained from pharmacies in İzmir with particular constraints [2]. The goal of basic, restricted and decomposition version of the variable neighbourhood search method is to find better solutions in the least amount of time by decomposing or restricting the search space. According to the findings, the basic and restricted versions of the method give better results significantly than the decomposition version of small and large scale instances. The customer utility maximization is a focal point in their research. The pharmacy duty scheduling problem is examined by adding special side constraints to a multi-period $p$ median model in [3]. Tabu search algorithm is used to solve the problem for the case in Izmir. They provide that the multiple duty pharmacy scheduling problem is $N P$-hard under the assumption of incapacitated pharmacies. The last study is on the location of pharmacies is belongs to [4]. They use set covering, $p$ center and $p$-median models with geographic information systems in order to select the most suitable triad of pharmacies -hospitals-warehouses to minimize the total transportation distance in the province of Gaziantep. They developed a GIS application to visualize the current and potential locations of the pharmacies.

Facility location is known as the most important starting point in the history of location theory [5]. The problems in this field refer to the several models each of which indicates different objectives and has different constraints. The applications of facility location spread to many areas such as emergency services, hospitals, fire stations, airline hubs, schools and warehouses among many others. In health care applications, the set covering model, maximal covering model and $p$ median model are taken attention more than the others [6].

The roots of MCLM were generalized in [1] and various extensions to the original problem have been made since then. A comprehensive literature review about the recent papers in the set covering model, maximal covering model and $p$-median model can be found in [7].

The basic maximal covering model aims to cover maximum demand with a fixed number of facilities. An extension of the model is proposed in [8]. They emphasize on the full and partial coverage of the demand nodes and lower level of service on the constraint of distance between the facility and the demand node. They mentioned on the behaviour of maximal covering model in which some demand points cannot be covered.

The model in [9] is a dynamic maximal covering model. They called the model dynamic because of the changing locations of the facilities in time steps in the whole period. It is the similar approach as considering opening some facilities in time steps in the period, while the others are closed.

Another extension of MCLM is formulated for different capacitated facilities. Several possible capacity levels of the facility at each potential site are used in contrast to only one fixed capacity level which limited the applications of a modular capacitated maximal covering location problem [10]. The article provided a discussion of modular capacitated maximal covering location problem with either facility constraint or non-facility constraint to determine the optimal location of ambulances.

Although the large scale MCLMs are difficult to solve, heuristics are useful to solve such kind of problems. The large-scale maximal covering problem is solved by genetic algorithms with up to 900 nodes in [11]. The results show that the proposed approach has a capacity to solve problems with up to 2500 nodes. A combination of fuzzy simulation and the greedy variable neighbourhood search is presented to solve large scale maximal coverage location problems in [12]. They use greedy VNS for searching solution space and fuzzy simulation to evaluate each solution obtained by greedy VNS. They solve the problem with 1000, 1500, and 2500 nodes and compare the results with the exact results obtained using CPLEX. MCLM maximize the coverage of population within a rapid nuclear response while minimizing modifications of the existing system [13]. They consider three phases including facility location, reallocation and coverage. At these phases, their first aim is to maximize the use 
of the existing system. The second is to determine the trade-offs between competing objectives, and the final one is to reduce the set of alternatives. After adding modified conditional covering problem constraints to the problem, they prevent facilities from covering nodes within close proximity in a worst-case scenario. Another variation of the maximal location coverage model is suggested in [14]. The location of facilities and allocation of customers are two main decision criteria of the problem which are considered in bi-level mathematical formulation. They propose greedy randomized adaptive search procedure (GRASP) and hybrid GRASP-Tabu search heuristics. The results of single level and bi-level formulation are compared based on convergence to optimality. It is determined that two heuristics can find good quality solutions.

As a contribution to the existing literature, besides the known objectives and constraints, the model is improved by adding distance restricted constraint for the special case of fairness between facilities in this study.

\section{Mathematical model}

A graph consists of a set of nodes and edges that refer to the location points and the lines connecting these location points. In this problem, the nodes represent demand points\&location of pharmacies and the edges represent distances between pharmacies and demand points. Nodes indexed by $i=1$....I, indicate the demand (site) points that are to be covered by pharmacies. Nodes indexed by $j=1 \ldots J$ indicate pharmacies that are candidates for covering the demand points on a given time period, $T$. The proposed model is an extension of MCLM. For the same reason that MCLM is NP-hard, our suggested model is also an NP-hard problem with three dimensional decision variables indexed respectively, $i, j$, and $t$. The model in this study is a mixed integer linear model and solved by using Lingo software which includes Simplex, branch and bound, and nonlinear solvers. We obtain feasible solution of the model, thanks to its small size.

\section{Distance restricted maximal covering location model (DR-MCLM) for pharmacy duty scheduling (PDS) problem:}

In this model, the distance between pharmacies on duty is restricted for each day of the period. The model tends to maximize the demands per distance covered by $p$ pharmacies in a given period $T$.

\section{Notations}

\section{Sets and Indices}

$i \in I$ : set of demand (site) points

$j, m \in J$ : set of facilities (pharmacies),

$t \in T$ : time periods (days),

$D=\left\{\mathrm{m}, j \in \mathcal{J} \mid s_{m j}<D R\right\}$

\section{Parameters}

$h_{i}$ : demand at node $i$.

$d_{i j}$ : distance between demand point $i$ and pharmacy $j$.

$s_{m j}$ : distance between pharmacy site $m$ and pharmacy $j$.

$n$ : number of pharmacies on duty in one period

$D R$ : maximum distance restricted between pharmacies on duty

M: a big number

$$
\begin{gathered}
y_{j t}=\left\{\begin{array}{l}
1, \text { if pharmacy } j \text { is on duty on day } t \\
0, \\
\text { otherwise }
\end{array}\right. \\
=\left\{\begin{array}{l}
1, \text { if demand point } i \text { is covered by pharmacy } j \\
0,
\end{array}\right. \\
\text { Maximize } \mathrm{Z}=\sum_{t=1}^{T} \sum_{j=1}^{J} \sum_{i=1}^{I} x_{i j t} h_{i} / d_{i j} \\
x_{i j t} \leq y_{j t} \\
\sum_{t=1}^{T} y_{j t}=1 \\
\sum_{j=1}^{J} x_{i j t} \leq 1 \\
\sum_{j=1}^{J} y_{j t} \geq n, t
\end{gathered}
$$$$
x_{i j t}=\left\{\begin{array}{lr}
1, \text { if demand point } i \text { is covered by pharmacy } j \text { on day } t \\
0, & \text { otherwise }
\end{array}\right.
$$

The objective function of the model represented by Eq. (1) aims to maximize the demand covered over the distance between site and pharmacies on duty for each day of period $T$. Eq. (2) prevents a site to be covered by a pharmacy which is not open on day $t$. Each pharmacy should be on duty once in the whole period is given in Eq. (3). Eq. (4) states that each site should not be covered more than once (no backup coverage) on each day. Eq. (5) ensures that at least $n$ number of the pharmacies must be on duty on each day. Eq. (6) is fairness constraint. It states that the distance between pharmacies on duty at the same day must not be less than the maximum distance restricted. As shown in the notations, the set $D$ is given including the index numbers of inconvenient pairs of pharmacies which can not have duty on the same day. Eq.(7) and Eq.(8) stand for the binary values of decision variables.

\section{Adana case}

In Adana case, the requirement of Adana Chamber of Pharmacists is to ensure fairness between pharmacies. In order to meet this fairness, Adana Chamber of Pharmacists emphasized that the distance between pharmacies on duty must not be less than $1.6 \mathrm{~km}$. It is mentioned by Adana Chamber of Pharmacists that the 
given pharmacies on the map are eligible to be on duty in terms of capacity and other requirements.

The model is run with data which were recorded in 2017 by Adana Chamber of Pharmacists. These data include the number of pharmacies and their locations over Çukurova. Çukurova is one of the biggest region in Adana and 114 pharmacies are located in this region. The Chamber of Pharmacists requires that the number of duty pharmacies must be at least 4 for this region in each day of the period. Therefore, the period include 28 days for this special situation.

On the map of Adana, 10 demand points with blue border lines are shown by their numbers in blue circles and the locations of pharmacies are given with their numbers in red symbols in Figure 1. One of the pharmacies with number 26 on the left side of the figure is located in the rural area of the city and it is also included in the model.

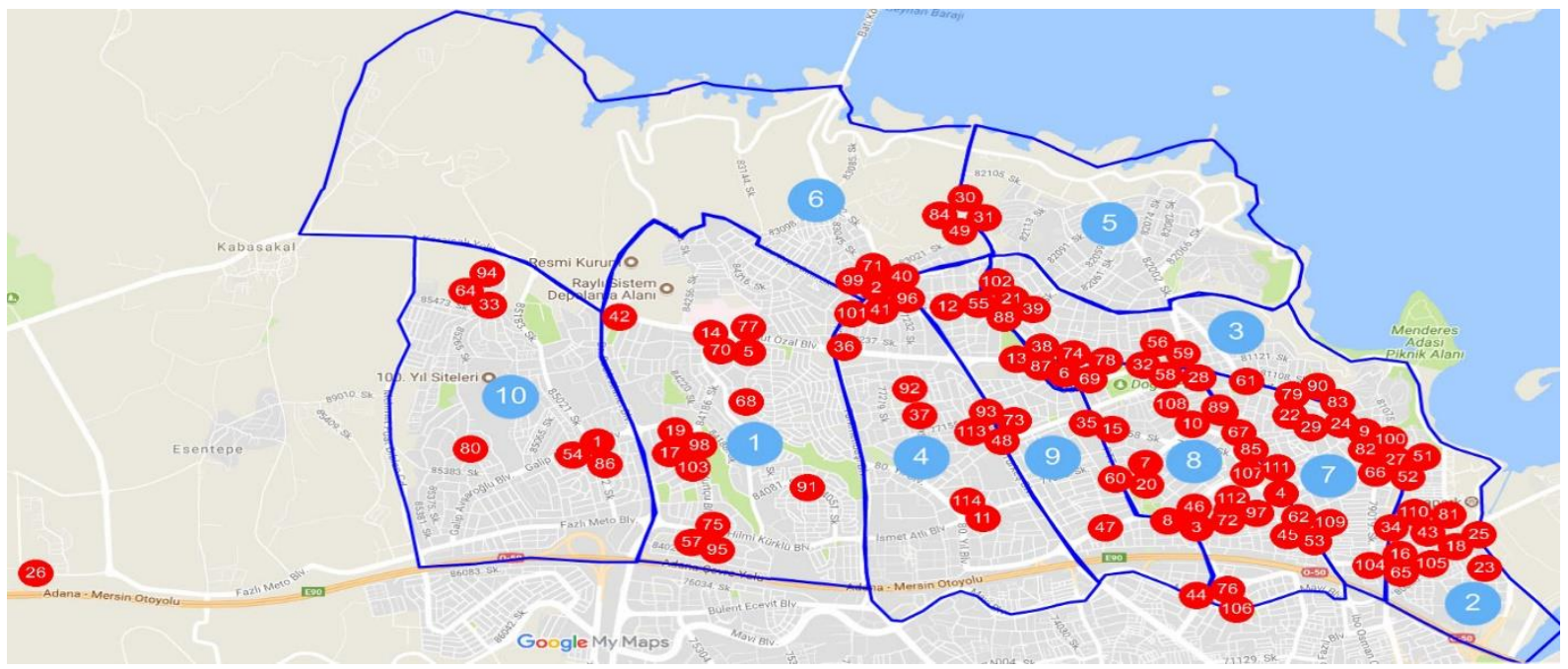

Figure 1. Sites and pharmacies located in the region of Çukurova.

\section{Results}

The distance restricted maximal covering location model is solved by using Lingo software with an Intel Core i7 $2.4 \mathrm{GHz}$ computer. The optimum result is reached after 24 minutes. The solution of DR-MCLM is difficult to be exhibited in one graph, because of its dimensions. Thus, the results are given in timely based graphs and four maps are used for the period of 28 days. Each graph shows a week and each day is shown by one colour. For each day, the points of locations of duty pharmacies are given within the same colour in Figure 2. It can be concluded that the model tends to choose the locations of pharmacies on duty not very close to each other $(\min 1.6 \mathrm{~km})$ while covering all the sites and maximizing the demand covered over distance.

The results show that the model finds the locations of duty pharmacies on each day of the period with the given constraints successfully. In Figure 2, each group of same coloured balls with the numbers of pharmacies are given for the same day of the period on the region.

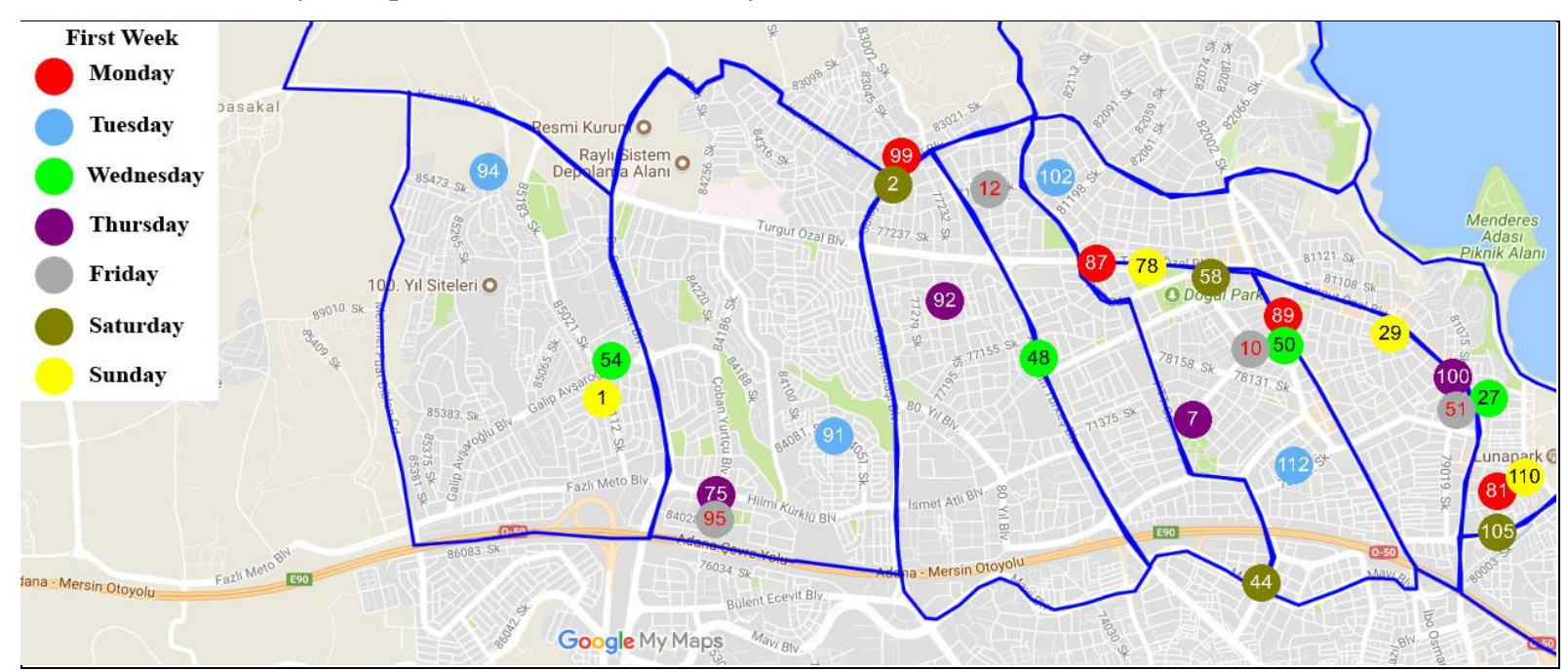



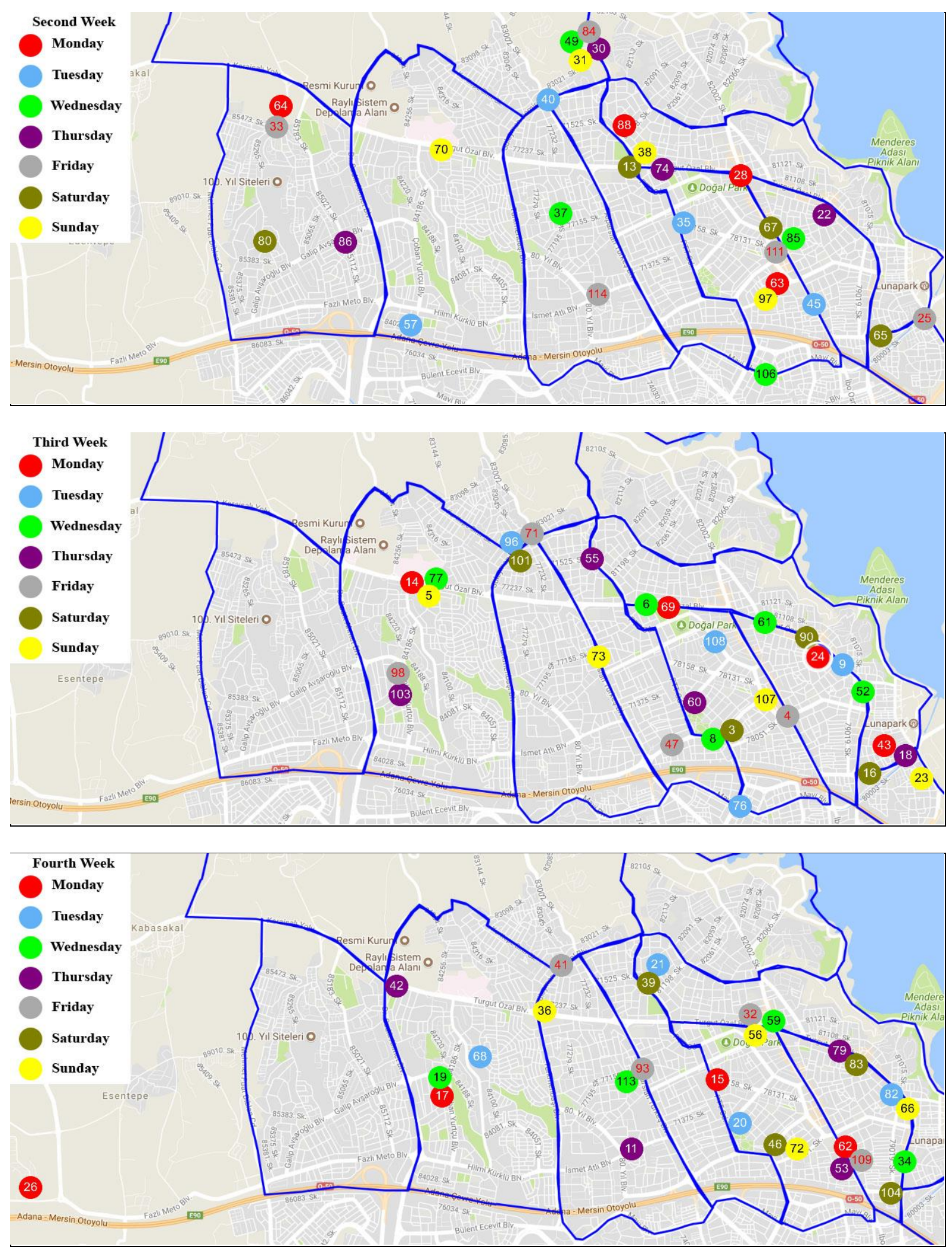

Figure 2. Duty pharmacies on each week in 28 days period.

The objective function value is attained approximately $1.64 \mathrm{E}+8$. This value is the maximum covered population over distances between pharmacies and the sites. This value is not a good indicator alone, however maximizing this value forces the model to assign the pharmacy to the nearest site with higher population.
The assignments of each site to duty pharmacy are demonstrated in Table 1 . The results can be analysed together with the Figures on the previous pages. All the sites are covered in each day of the period and the results seem fair. 
Table 1. Weekly representation of covered sites by duty pharmacies.

\begin{tabular}{|c|c|c|c|c|c|c|c|}
\hline \multicolumn{2}{|c|}{ 1st Week } & \multicolumn{2}{|c|}{ 2nd Week } & \multicolumn{2}{|c|}{ 3th Week } & \multicolumn{2}{|c|}{ 4th Week } \\
\hline $\begin{array}{l}\text { Pharmacy } \\
\text { No }\end{array}$ & $\begin{array}{l}\text { Covered } \\
\text { Sites }\end{array}$ & $\begin{array}{c}\text { Pharmacy } \\
\text { No }\end{array}$ & $\begin{array}{l}\text { Covered } \\
\text { Sites }\end{array}$ & $\begin{array}{c}\text { Pharmacy } \\
\text { No }\end{array}$ & $\begin{array}{l}\text { Covered } \\
\text { Sites }\end{array}$ & $\begin{array}{c}\text { Pharmacy } \\
\text { No }\end{array}$ & $\begin{array}{l}\text { Covered } \\
\text { Sites }\end{array}$ \\
\hline 81 & 2,7 & 28 & 3,9 & 14 & 6,10 & 15 & $1,3,4,5,9$ \\
\hline 87 & $1,4,5$ & 63 & $2,4,7,8$ & 24 & 3,8 & 17 & 10 \\
\hline 89 & $3,8,9$ & 64 & 10 & 43 & 2,7 & 26 & 6 \\
\hline 99 & 6,10 & 88 & $1,5,6$ & 69 & $1,4,5,9$ & 62 & $2,7,8$ \\
\hline 91 & 1,10 & 35 & $1,3,4,5,9$ & 9 & 7 & 20 & $3,4,8,9$ \\
\hline 94 & 6 & 40 & 6 & 76 & 2 & 21 & 5,6 \\
\hline 102 & 5 & 45 & $2,7,8$ & 96 & $1,6,10$ & 68 & 1,10 \\
\hline 112 & $2,3,4,7,8,9$ & 57 & 10 & 108 & $3,4,5,8,9$ & 82 & 2,7 \\
\hline 27 & 2,7 & 37 & $1,4,10$ & 6 & 1,5 & 19 & 6,10 \\
\hline 48 & $1,4,5,6$ & 49 & 5,6 & 8 & $4,8,9$ & 34 & $2,7,8$ \\
\hline 50 & $3,8,9$ & 85 & $3,7,8,9$ & 52 & 2,7 & 59 & $3,5,9$ \\
\hline 54 & 10 & 106 & 2 & 61 & 3 & 113 & 1,4 \\
\hline & & & & 77 & 6,10 & & \\
\hline 7 & $3,4,8,9$ & 22 & $2,3,7,8$ & 18 & 2,7 & 11 & 1,4 \\
\hline 75 & 10 & 30 & 6 & 55 & $1,5,6$ & 42 & 6,10 \\
\hline 92 & $1,5,6$ & 74 & $1,4,5,9$ & 60 & $3,4,8,9$ & 53 & $2,7,8$ \\
\hline 100 & 2,7 & 86 & 10 & 103 & 10 & 79 & $3,5,9$ \\
\hline 10 & $3,4,8,9$ & 25 & 2 & 4 & $2,3,7,8$ & 32 & $3,5,9$ \\
\hline 12 & $1,5,6$ & 33 & 10 & 47 & $1,4,9$ & 41 & 6,10 \\
\hline 51 & 2,7 & 84 & 5,6 & 71 & 5,6 & 93 & 1,4 \\
\hline 95 & 10 & 111 & $3,7,8,9$ & 98 & 10 & 109 & $2,7,8$ \\
\hline & & 114 & 1,4 & & & & \\
\hline 2 & $1,6,10$ & 13 & $1,4,5,6$ & 3 & $4,8,9$ & 39 & $1,5,6,10$ \\
\hline 44 & 8 & 65 & 2,7 & 16 & 2,7 & 46 & $4,8,9$ \\
\hline 58 & $3,4,5,9$ & 67 & $3,8,9$ & 90 & 3 & 83 & 3 \\
\hline 105 & 2,7 & 80 & 10 & 101 & $1,5,6,10$ & 104 & 2,7 \\
\hline 1 & 10 & 31 & 6 & 5 & 6,10 & 36 & $1,6,10$ \\
\hline 29 & 3,8 & 38 & 1,5 & 23 & 2,5 & 56 & $3,5,9$ \\
\hline 78 & $1,4,5,6,9$ & 70 & 10 & 73 & 1,4 & 66 & 2,7 \\
\hline 110 & 2,7 & 97 & $2,3,4,7,8,9$ & 107 & $3,7,8,9$ & 72 & 4,8 \\
\hline
\end{tabular}

\section{Conclusion}

A distance restricted maximal covering model for the problem of pharmacies duty scheduling is provided in this study. The special case of the problem is the distance restriction that has to be considered between pharmacies on duty. For the classical MCLM, the demand point is assumed to be covered completely if 
located within the critical distance of the facility and not covered at all outside of the critical distance. Most of the models in location science consider the distances between facility and the sites. Comparing with classical MCLM, the contribution to the model lies in the critical distance between the facilities taken into account in this study. We emphasized on this critical distance between pharmacies and fortunately this distance does not occur as an infeasibility for our case. The constraints for distances may result an infeasibility, because of the reason that the real system may not been established under this constraint at the beginning. The feasibility may not be reachable case to case depend on the given data. Therefore, the model proposed in this study need to be solved by heuristics or relaxation methods not by exact methods for some data related to large systems, if required. Thanks to the size of the problem and appropriate data in our case, the optimal duty schedules and assignments of demand points are obtained by using Lingo software. The results are found reasonable by the Chamber of Pharmacists based on its applicability, but they mentioned that the patients cannot be restricted to get medicine from an assigned pharmacy obtained by our results, as it is also obvious for us. On the other hand, the assignment of demand points to pharmacies allows to balance the demand over the facilities while scheduling the duties. For further studies, the suggested model could be extended to the whole city in the same case in order to compare the whole schedule in practice. Therefore, the performance of proposed model can be investigated up to a large number of sites and pharmacies.

\section{Acknowledgements}

The authors would like to thank Adana Chamber of Pharmacists for providing data used in this manuscript and the editors of this journal and anonomys reviewers for their valuable comments.

\section{References}

[1] Church, R., \& ReVelle, C. (1974). Maximal covering location problem. Papers of the Regional Science Association. 32, 101-118.

[2] Kocatürk, F., \& Özpeynirci, Ö. (2014). Variable neighborhood search for the pharmacy duty scheduling problem. Computers \& Operations Research. 51, 218-226.

[3] Kocatürk, F., \& Ağlamaz, E. (2016). Pharmacy duty scheduling problem. International Transactions in Operational Research. 23, 459-480.

[4] Özceylan, E., Uslu, A., Erbaş, M., Çetinkaya, C., \& İşleyen, S.K. (2017). Optimizing the locationallocation problem of pharmacy warehouses: A case study in Gaziantep. An International Journal of Optimization and Control: Theories \& Applications. 7(1), 117-129.

[5] Farahani, R.Z., SteadieSeifi, M. \& Asgari, N. (2010). Multiple criteria facility location problems:
A survey. Applied Mathematical Modelling. 34, 1689-1709.

[6] Daskin, M. S., \& Dean, L. K. (2004). Location of Health Care Facilities. Chapter 3 in the Handbook of OR/MS in Health Care: A Handbook of Methods and Applications. Kluwer.

[7] ReVelle, C.S., Eiselt, H.A., \& Daskin, M.S. (2008). A bibliography for some fundamental problem categories in discrete location science. European Journal of Operational Research, 184, 817-848.

[8] Karasakal, O., \& Karasakal, E.K. (2004). A maximal covering location model in the presence of partial coverage. Computers \& Operations Research, 31, 1515-1526.

[9] Zarandi, M.H.F., Davari, S., \& Sisakht, S.A.H. (2013). The large-scale dynamic maximal covering location problem. Mathematical and Computer Modelling, 57, 710-719.

[10] Yin, P., \& Mu, L., (2012). Modular capacitated maximal covering location problem for the optimal siting of emergency vehicles. Applied Geography, 34, 247-254.

[11] Zarandi ,M.H.F., Davari, S., \& Sisakht, S.A.H. (2011). The large scale maximal covering location problem. Scientia Iranica, 18(6), 1564-1570.

[12] Davari, S., Zarandi ,M.H.F., \& Turksen, I.B. (2013). A greedy variable neighborhood search heuristic for the maximal covering location problem with fuzzy coverage radii. Knowledge-Based Systems, 41, 6876.

[13] Paul, N.R., Lunday, B.J., \& Nurre, S.G. (2017). A multiobjective, maximal conditional covering location problem applied to the relocation of hierarchical emergency response facilities. Omega, 66, 147-158.

[14] Diaz, J.A., Luna, D.E, Vallejo, J-F.C., \& Ramirez, M-S.C. (2017). GRASP and hybrid GRASP-Tabu heuristics to solve a maximal covering location problem with customer preference ordering. Expert Systems with Applications, 82, 62-76.

Nuşin Uncu received her PhD from Department of Industrial Engineering in Cukurova University. She is working as an Assistant Professor in Adana Science and Technology University. She received a scholarship granted by TUBITAK for postdoctoral studies on process mining in Eindhoven University of Technology in 2017. Her research area includes modelling, analyzing and mining of complex systems and processes.

Berna Bulğurcu received her PhD from Department of Business in Çukurova University. She is working as an Assistant Professor in Çukurova University. Her research interests are multiobjective optimization methods and quantitative decision methods. 
Fatih Kulıç received his PhD from Department of Electrical\&Electronics Engineering in Cukurova University. He is working as an Assistant Professor in Adana Science and Technology University. His research area includes public transit network and search algorithms.

An International Journal of Optimization and Control: Theories \& Applications (http://ijocta.balikesir.edu.tr)

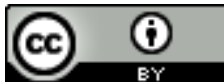

This work is licensed under a Creative Commons Attribution 4.0 International License. The authors retain ownership of the copyright for their article, but they allow anyone to download, reuse, reprint, modify, distribute, and/or copy articles in IJOCTA, so long as the original authors and source are credited. To see the complete license contents, please visit http://creativecommons.org/licenses/by/4.0/. 